\title{
An update on neuroanesthesia for the occasional neuroanesthesiologist
}

Piyush Patel MD

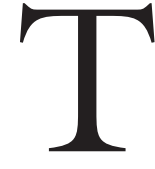

HE anesthetic management of neurosurgical patients is, by necessity, based upon our understanding of the physiology and pathophysiology of the central nervous system (CNS) and the effect of anesthetic agents on the CNS. Consequently, a great deal of investigative effort has been expended to elucidate the influence of anesthetics on CNS physiology and pathophysiology. The current practice of neuroanesthesia is based upon findings of these investigations. However, it should be noted that most studies in this field have been conducted in laboratory animals and the applicability of the findings to the human patient is debatable at best. A great deal of emphasis has been placed on the minor differences in anesthetic-induced changes in cerebral blood flow $(\mathrm{CBF})$, cerebral metabolic rate $(\mathrm{CMR})$ and intracranial pressure (ICP) that have been consistently demonstrated in a variety of studies. Is this emphasis justified? It is not surprising that, in the absence of controlled studies which demonstrate the superiority of one technique over another, interpretations of the available data differ and opinions on the optimal approach to the neurosurgical patient also differ. A more important question to the practicing anesthesiologist is not whether the minor differences in CNS physiology induced by anesthetics are relevant to all neurosurgical patients, but the identification of clinical situations in which anesthetic effects might be significant.

In the present discussion, a brief review of the cerebrovascular effects of anesthetic agents will be presented. Thereafter, situations in which the anesthetic selection has been suggested to be relevant will be addressed:

1) Moderate to severe intracranial hypertension $(\mathrm{ICH})$

2) Inadequate brain relaxation during surgery

3) Evoked potential monitoring

4) Intraoperative electrocorticography

5) Cerebral protection

\section{CNS effects of anesthetic agents}

It is now generally accepted that $\mathrm{N}_{2} \mathrm{O}$ is a cerebral vasodilator which can increase $\mathrm{CBF}$ when administered alone. This vasodilation can result in an increase in ICP. In addition, $\mathrm{N}_{2} \mathrm{O}$ can also increase CMR to a small extent. The simultaneous administration of in anesthetics (barbiturates, propofol, benzodiazepines, narcotics) can substantially reduce this increase in $\mathrm{CBF}$ and $\mathrm{CMR}$. The behaviour of a combination of volatile agents and $\mathrm{N}_{2} \mathrm{O}$ is quite different. When administered in low doses, volatile agents can reduce $\mathrm{CBF}$ and CMR. The addition of $\mathrm{N}_{2} \mathrm{O}$ to low dose volatile agent anesthesia increases both $\mathrm{CBF}$ and CMR. This $\mathrm{N}_{2} \mathrm{O}$ mediated vasodilation can be greater when higher doses of volatile agents are administered.

Volatile agents uniformly suppress CMR. At doses of 1.5 to 2.0 minimum alveolar concentration (MAC), the commonly used agents isoflurane, desflurane and sevoflurane all produce burst suppression of the electroencephalograhy (EEG). At burst suppression, CMR is reduced by 50 to $60 \%$. Volatile agents are also vasodilators. Their effect on CBF is biphasic. At doses of about 0.5 MAC, the suppression in CMR balances the vasodilatory effects and CBF does not change significantly. In doses greater than 1.0 MAC, the vasodilatory effect predominates and $\mathrm{CBF}$ increases. The addition of $\mathrm{N}_{2} \mathrm{O}$ to volatile-based anesthesia will increase $\mathrm{CBF}$ and CMR. This increase in CBF may not necessarily result in an increase in ICP. Of greater importance than changes in $\mathrm{CBF}$ are changes in cerebral blood volume (CBV). About 65 to $70 \%$ of intracranial blood resides in the veins and sinuses. While the effect of volatile agents on CBV parallel the CBF, these changes are of a significantly lesser magnitude. For example, sevoflurane results in a substantially lesser $\mathrm{CBF}$ increase than does an equivalent dose of isoflurane. However, the effect of these agents on CBV is not different. Propofol, on the other hand, reduces both $\mathrm{CBF}$ and $\mathrm{CBV}$.

Intravenous hypnotic agents, with the exception of ketamine, all decrease CMR and CBF substantially.

From the Department of Anesthesia, VA Medical Center, University of California, San Diego, California, USA.

Address correspondence to: Dr. Piyush Patel, Anesthesia Service 125, VA Medical Center, 3350 La Jolla Village Drive, San Diego, CA

92161, USA. Phone: 858-552-8585, ext. 6927; Fax: 858-534-0104; E-mail: ppatel@ucsd.edu 
Propofol has also been shown to significantly decrease CBV. In appropriate doses, barbiturates, propofol and etomidate produce burst suppression of the EEG. Ketamine's effect on $\mathrm{CBF}$ and CMR are regionally specific; in the limbic structures, $\mathrm{CBF}$ and $\mathrm{CMR}$ increase whereas within the cortex, reductions in $\mathrm{CBF}$ and CMR occur.

In terms of vasodilation potency, desflurane produces the greatest amount of vasodilation, followed by isoflurane and sevoflurane; propofol, etomidate and the barbiturates effect vasoconstriction.

\section{1) Moderate to severe $\mathrm{ICH}$}

Patients with ICH have symptoms of headache, nausea, vomiting and visual disturbance. Patients with severe ICH also have a reduced level of consciousness. Computed tomography scans demonstrate mass lesions, ventricular effacement, midline shifts of the brain and full basal cisterns. The brain's capacity to accommodate increases in CBV is exhausted and even slight increases in intracranial volume can result in dramatic increases in ICP. In patients with acute increases in ICP (for example, with traumatic brain injury, epidural and subdural hematomas), the effect of an increase in CBV on ICP is even greater. It is in these patients that the choice of anesthetic agents must be considered carefully.

Agents that produce vasodilation can increase CBF and more importantly, CBV. The potential for a further increase in ICP is therefore apparent. Minor increases in ICP can be treated readily by modest hyperventilation and the use of diuretics. Consequently, for the majority of patients, it is unlikely that anesthetic induced increases in ICP will be substantial enough to compromise the brain. For example, in patients with intracranial tumours, there were no differences in outcome in patients anesthetized with propofol-fentanyl, isoflurane- $\mathrm{N}_{2} \mathrm{O}$ or fentanyl-nitrous oxide. ${ }^{1}$ Nonetheless, other studies have shown that dural tension is higher with isoflurane-fentanyl and sevoflurane-fentanyl anesthesia in comparison to propofol-fentanyl anesthesia. ${ }^{2}$ For patients in whom the ability of the brain to compensate for further increases in CBV is exhausted, a technique that reduces CMR, CBV and ICP may be preferable. In such patients, it is the author's practice to avoid $\mathrm{N}_{2} \mathrm{O}$ and volatile agents until such time as the dura is opened. An anesthetic technique based on the infusion of propofol and opioids may be a more prudent approach in so far as the reserve of the brain to compensate for increases in $\mathrm{CBV}$ is not encroached upon and may in fact be increased (reduction in CBV). Volatile agents may be introduced once the cranium has been opened and the dura has been reflected; observa- tion of the brain and the surgical conditions can then dictate the anesthetic regimen.

A similar logic may apply to the management of the acutely head injured patient. Compensatory mechanisms are inadequate to offset the rapid increase in intracranial volume and ICP. In such patients, brain distortion and herniation can compromise regional brain perfusion, rendering the brain ischemic. Moreover, experimental data have shown that hyperventilation, which is often employed to minimize or counteract volatile agent induced vasodilation, can be ineffective in doing so with acute head injury. ${ }^{3} \mathrm{~A}$ cogent argument can therefore be made that one should avoid $\mathrm{N}_{2} \mathrm{O}$ and volatile agents in the anesthetic management.

\section{2) "Tight brain" during surgery}

Adequate brain relaxation facilitates neurosurgery and reduces the need for excessive brain retraction. Although uncommon, brain swelling can occur intraoperatively during surgery. This is most commonly seen during arteriovenous malformation surgery, but it can occur during tumour surgery as well. The etiology of brain swelling is not clear. Clearly, engorgement of the brain with blood plays a significant role. When brain swelling does occur, the brain is placed at risk for ischemic injury. In addition, brain swelling interferes with surgery and on occasion, can prevent closure of the dura. This represents an urgent problem that demands the attention of the anesthesiologist and the neurosurgeon. The approach to this difficult problem is reasonably well established and the following maneuvers may be instituted:

- Check ventilation. Moderate hypocapnia (target $\mathrm{PaCO}_{2}$ 25-30 mmHg) will produce cerebral vasoconstriction and consequent reduction in brain bulk. Measurement of end-tidal $\mathrm{CO}_{2}$ tension is occasionally misleading. Arterial blood gas analysis should be utilized judiciously to confirm hypocarbia.

- Ensure normal oxygenation.

- Control blood pressure. Target is normotension (within 10\% of baseline blood pressure).

- Ensure adequate venous drainage from the brain. Neck torsion or the placement of endotracheal tube ties around the neck can impede venous drainage from the brain.

- Head elevation $\left(30^{\circ}\right.$ optimum).

- Check intrathoracic pressure. Rule out pneumothorax (especially if central line has been placed).

- Maintain adequate neuromuscular relaxation.

- Administer mannitol. 
If these measures are not adequate, then consideration should be given to the potential deleterious effect of anesthetic agents. In particular, attention should be focused on those agents that can increase brain bulk by producing cerebral vasodilation. The manipulation of anesthetic administration can effect dramatic reductions in brain bulk:

- Make sure that the concentration of volatile agent is less than 0.5 MAC

- Discontinue the administration of $\mathrm{N}_{2} \mathrm{O}$

- Discontinue the administration of volatile anesthetics

- Switch to an iv anesthetic technique. A combination of propofol and opioid infusion is ideal.

- If the brain swelling does not abate, then the probability that the patient will have protracted $\mathrm{ICH}$ in the postoperative period is high. In that event, barbiturates (pentobarbital) may be administered until either the swelling is reduced or until burst suppression of the EEG is attained. On rare occasions, the surgeons may elect to amputate brain or to close the scalp without replacing the bone flap.

\section{3) Inadequate signal quality during evoked poten- tial monitoring}

Somatosensory evoked potentials (SSEP)

All volatile agents attenuate evoked potentials in a dose related manner (see an excellent review by Banoub et al.). ${ }^{4}$ SSEP amplitude can be attenuated at 1.0 MAC concentrations and can be abolished with higher concentrations. Simultaneously, a dose dependent increase in latency is also observed. The newer volatile agents sevoflurane and desflurane appear to depress the amplitude of the SSEP to a lesser extent and their use may permit the delivery of a higher concentration ( 1-1.5 MAC). ${ }^{5}$ Auditory evoked potentials are relatively robust but even their waveforms will be affected at volatile anesthetic concentrations that exceed 1.5 MAC. $\mathrm{N}_{2} \mathrm{O}$ can also reduce the amplitude of the SSEP. ${ }^{6}$ Intravenous agents, on the other hand, have a modest impact on evoked potentials; in fact, evoked potentials can be detected even with doses of barbiturates that produce burst suppression of the EEG.

Given that anesthetic agents suppress evoked potentials, the choice of anesthetic agents for the maintenance of anesthesia becomes an important consideration. Although volatile agents and $\mathrm{N}_{2} \mathrm{O}$ suppress evoked potentials, stable and robust evoked potential recordings can be obtained provided the concentration of the volatile agent is kept to 0.5 MAC or less and the $\mathrm{N}_{2} \mathrm{O}$ concentration is maintained in the 50 to $60 \%$ range. Without $\mathrm{N}_{2} \mathrm{O}$, the volatile anesthetic con- centration can be increased to about 1 MAC. Opioid infusion in addition will provide, in most circumstances, stable anesthetic conditions that permit evoked potential monitoring. If the quality of the signals is not adequate, then the anesthetic technique has to be modified. The technique that results in a very good signal is a total $i v$ anesthetic technique. The combination of propofol and an opioid infusion results in excellent signals in most patients. ${ }^{7}$ In addition, the variability in the amplitude of the evoked potential is reduced by this technique in comparison to a $\mathrm{N}_{2} \mathrm{O}$-volatile agent-narcotic technique. This is an important consideration in those patients with CNS abnormalities in whom the evoked potential is already compromised by the primary disease. If the signal does not improve, then it is highly unlikely that the cause of the problem is the anesthetic.

Etomidate is unique among anesthetic agents in that it actually increases the amplitude of SSEP. Clinicians often administer etomidate by infusion to improve the quality of the recording. However, it is difficult to determine what exactly an improvement in the signal that is induced by etomidate means to the transmission of the signal from the peripheral nerve to the brain. In addition, the usual criteria for determining a change in the evoked potential (amplitude reduction by $50 \%$ and latency delay by $10 \%$ ) may not apply when etomidate is administered. Nonetheless, in patients with significant sensory abnormalities, an anesthetic technique based on an etomidate infusion may be considered. Such a technique may allow reasonable evoked potential recordings which otherwise may not be possible. ${ }^{8}$

\section{Motor evoked potentials (MEP)}

MEP monitoring is a relatively new technique that is being increasingly employed during spine surgery that entails a significant risk of injury to the motor tracts. In many instances, the ability to reliably monitor the motor tracts has replaced the intraoperative wake-up test. In the operating room, transcranial electrical rather than magnetic stimulation, applied to the scalp, is used to depolarize cortical pyramidal tracts and to evoke a motor response in the upper and lower extremities. MEP are exquisitely sensitive to anesthetic agents. Volatile agents (in concentrations as low as 0.2-0.3 MAC), barbiturates, propofol and midazolam all significantly suppress MEP. ${ }^{9,10}$ It is therefore apparent that the anesthetic technique for MEP monitoring has to be substantially modified. In addition, the administration of muscle relaxants has to be strictly titrated to ensure that muscle contraction in the monitored limb is possible. ${ }^{11}$ Etomidate, $\mathrm{N}_{2} \mathrm{O}$, narcotics 
and ketamine are all reasonable agents to use. In the author's institution, the following technique has been successful:

Premedication: diazepam, $0.1 \mathrm{mg} \cdot \mathrm{kg}^{-1} 30$ to 60 min prior to anesthesia induction;

Induction: sufentanil $\mathrm{l} \mu \mathrm{g} \cdot \mathrm{kg}^{-1}$, etomidate 0.3 $\mathrm{mg} \cdot \mathrm{kg}^{-1}$;

Muscle relaxation: vecuronium or rocuronium, administered by a servo controlled loop mechanism that maintains the $\mathrm{Tl}$ twitch height at $35 \%$ of baseline twitch height;

Maintenance: $65 \% \mathrm{~N}_{2} \mathrm{O}$ in oxygen, sufentanil infusion at 0.4 to $0.5 \mu \mathrm{g} \cdot \mathrm{kg}^{-1} \cdot \mathrm{hr}^{-1}$;

Maintenance: etomidate infusion 5 to 10 $\mu \mathrm{g} \cdot \mathrm{kg}^{-1} \cdot \mathrm{min}^{-1}$ after load of $0.1 \mathrm{mg} \cdot \mathrm{kg}^{-1}$. Consider if additional anesthetic is deemed necessary or if use of $\mathrm{N}_{2} \mathrm{O}$ is not feasible (i.e., one lung ventilation).

The recent introduction of a multiple stimulation device has simplified to some extent the anesthetic management. Multiple stimuli, from 2 to 5 , with about 75 msec intervals between the stimuli, significantly improves the amplitude of the MEP. Moreover, this MEP is less susceptible to anesthetic induced suppression. ${ }^{12}$ Accordingly, low doses of volatile agent ( 0.3 MAC), propofol- $\mathrm{N}_{2} \mathrm{O}$, propofol-remifentanil ${ }^{13}$ and isoflurane- $\mathrm{N}_{2} \mathrm{O}$-opioid ${ }^{14}$ techniques may be compatible with adequate MEP monitoring. However, it should be noted that the administration of isoflurane reduces the percentage of patients in whom reliable MEP recording is possible and it increases the variability in the amplitude of the MEP. Accordingly, it may be prudent to establish robust MEP monitoring before volatile agents are added to the anesthetic regimen.

\section{4) Intraoperative electrocorticography (ECoG)}

In patients undergoing craniotomy for resection of seizure producing foci, intraoperative ECoG is often employed. ECoG is used to precisely identify the location of the lesion and the margins of safe brain resection. Seizure foci are identified by characteristic spike waves that are elicited by electrical stimulation of the surrounding brain region. Once the foci are identified, they are removed. ECoG is then used to confirm the removal of the relevant focus - a lack of spike waves will confirm this.

Epilepsy surgery can be performed in an awake or anesthetized patient. Awake craniotomy is usually reserved for cooperative adult patients. Uncooperative patients or pediatric patients are generally anesthetized for the procedure.

During awake craniotomy, patients are usually sedated with an infusion of propofol during the craniotomy. Thereafter, the propofol infusion is discontin- ued and the patient is allowed to awaken. Upon resumption of consciousness, ECoG mapping is started. Propofol is an ideal agent to use because its pharmacokinetic properties permit rapid emergence from a state of anesthesia. However, it should be remembered that propofol can have a profound effect on the ECoG. Residual propofol in the brain can result in EEG activation in the $18 \mathrm{~Hz}$ range. ${ }^{15}$ This activation can obscure spike waves from the seizure foci, thereby making precise localization of the foci difficult. The EEG activation can occur even when the patient appears to be fully awake! EEG activation is generally short lived and lasts about $20 \mathrm{~min}$. It is therefore important to discontinue the administration of propofol at least $30 \mathrm{~min}$ before the start of ECoG. More recently, the addition of remifentanil to a propofol infusion has permitted a reduction in the dose of propofol; a more rapid emergence (within ten minutes) is therefore possible. Anesthetics that suppress seizure foci (benzodiazepine, volatile agents) should, in general, be avoided.

ECoG during general anesthesia is more challenging. Volatile anesthetics, iv hypnotics and benzodiazepines can suppress spike waves. Therefore, during ECoG, the use of these agents must be minimized or avoided altogether. An anesthetic technique that is commonly used for this procedure is a combination of $\mathrm{N}_{2} \mathrm{O}$, low dose volatile agent and an opioid infusion. Shortly before ECoG, the volatile agent is discontinued and the concentration of $\mathrm{N}_{2} \mathrm{O}$ is increased to at least $65 \%$. Once the volatile agent is eliminated, ECoG can be performed. During this time, the patient will be lightly anesthetized and there is a significant risk of movement or coughing. It is therefore very important to ensure that the patient is paralyzed. If the spike waves are not detectable, spike activity can be induced by the administration of one of the following:

i) Methohexital, 0.3 to $0.5 \mathrm{mg} \cdot \mathrm{kg}^{-1} \cdot{ }^{16}$ Methohexital results in spike wave activity that emanates primarily from the seizure focus.

ii) Etomidate, 0.1 to $0.2 \mathrm{mg} \cdot \mathrm{kg}^{-1} \cdot{ }^{17}$ The resulting spike wave activity is more generalized that with methohexital.

iii) Alfentanil, $50 \mathrm{~g} \cdot \mathrm{kg}^{-1} \cdot{ }^{18}$

\section{5) Brain protection}

There is a considerable risk of cerebral ischemia during neurosurgical procedures and a substantial investigative effort has focused upon the identification of agents that might reduce ischemic brain injury. Given their propensity to reduce CMR, anesthetics appear to be logical candidates. Volatile agents, barbiturates, propofol and ketamine have all shown neuroprotective 
efficacy in the laboratory. Unfortunately, this neuroprotection is short lived and is not sustained beyond a period of two weeks. ${ }^{19}$ Neurons continue to die for a long time after the initial ischemic insult and anesthetics may mitigate ongoing neuronal loss. In the circumstance of extremely mild ischemic insults, such as those that are likely to occur with brain retraction, anesthetics might produce sustained neuroprotection. However, with such mild insults, it is highly unlikely that differences in the neuroprotective efficacy for individual anesthetics will be manifest. Accordingly, the available data do not support the selection of any given agent for purposes of neuroprotection. Adequate anesthesia, regardless of how it is produced, will increase the tolerance of the brain to ischemia in comparison to the awake state. Barbiturates may be used for purposes of neuroprotection in rare situations (such as prolonged temporary clipping during aneurysm surgery).

Among iv hypnotics, the only drug whose use might be considered controversial is etomidate. Etomidate can reduce CMR, CMF and ICP. These effects are similar to those produced by barbiturates and propofol. Unlike the latter drugs, etomidate does not produce hypotension. Given this favourable pharmacologic profile, the use of etomidate during neurosurgical procedures that entail a risk of cerebral ischemia has been advocated. Unfortunately, it is quite clear from laboratory studies and studies in patients ${ }^{20}$ that etomidate does not possess neuroprotective efficacy. In fact, it can increase ischemic neuronal injury. The administration of etomidate for the purposes of neuroprotection therefore cannot be advocated. Single doses of etomidate for purposes of anesthetic induction are unlikely to adversely affect neurons and its use in this manner is entirely appropriate.

\section{6) Toxicity}

The administration of sevoflurane to patients who have a pre-existing seizure disorder has been shown to result in EEG evidence of seizure activity. Seizure activity has been demonstrated to occur in clinically applicable doses. To date, seizures have not been observed with isoflurane or desflurane anesthesia. A more recent report indicated that seizures can occur even in otherwise healthy individuals who have no prior medical history of epilepsy. ${ }^{21}$ This pro-convulsant effect of sevoflurane is dose dependent. However, seizures attributable to sevoflurane administration have also been noted on emergence after uneventful general anesthesia.

Of greater concern to anesthesiologists is recent evidence which suggests that in neonatal rat pups, anesthetic agents can actually lead to neuronal death. In this limited experimental model, neuronal injury is caused by apoptosis. Exposure to a variety of agents, including volatile anesthetics, barbiturates, benzodiazepines, $\mathrm{N}_{2} \mathrm{O}$ and ketamine, can result in neuronal apoptosis. ${ }^{22}$ This injury is manifest as a reduction in cognitive ability of rats once they survive to adulthood. Although the mechanism by which this injury is produced is not known, an imbalance between GABA mediated inhibition and NMDA antagonism is thought to play a central role. ${ }^{23}$ The critical period of vulnerability appears to be the time during which synaptogenesis is active. The relevance of these laboratory findings to the clinical use of anesthetic agents is not clear at the moment. A recent study, published in abstract form, indicated that anesthetics did not cause neuronal injury in animal models in which the period of synaptogenesis was considerably longer than in rodents. Synaptogenesis in humans extends over several years after birth and whether a brief anesthetic exposure will result in neuronal death is not known. Given that almost all anesthetics in use today either enhance inhibition or antagonize excitation, there is little that the clinician can do to minimize the damage (if indeed such damage occurs in humans).

\section{Summary}

In the vast majority of neurosurgical patients, the choice of anesthetic agent is not relevant; the choice of anesthetic agents is unlikely to affect either the surgical field or the patient outcome. The best results are often obtained by the use of a technique with which the anesthesia care provider is familiar. In certain situations, however, the choice of the anesthetic agent can directly impact the surgical field and may have an impact on patient outcome. It is during these situations that a solid command of physiology and the pathophysiology of the CNS and the impact that anesthetic agents have on the brain is essential.

\section{References}

1 Todd MM, Warner DS, Sokoll MD, et al. A prospective, comparative trial of three anesthetics for elective supratentorial craniotomy: propofol/fentanyl, isoflurane/nitrous oxide, and fentanyl/nitrous oxide. Anesthesiology 1993; 78: 1005-20.

2 Petersen KD, Landsfeldt U, Cold GE, et al. Intracranial pressure and cerebral hemodynamic in patients with cerebral tumors. Anesthesiology 2003; 98: 329-36.

3 Scheller MS, Todd MM, Drummond JC. A comparison of the ICP effects of isoflurane and halothane after cryogenic brain injury in rabbits. Anesthesiology 1987; 67: 507-12. 
4 Banoub M, Tetzlaff JE, Schubert A. Pharmacologic and physiologic influences affecting sensory evoked potentials: implications for perioperative monitoring. Anesthesiology 2003; 99: 716-37.

5 Bernard JM, Pereon $\Upsilon$, Fayet G, Guibeneuc P. Effects of isoflurane and desflurane on neurogenic motor- and somatosensory evoked potential monitoring for scoliosis surgery. Anesthesiology 1996; 85: 1013-9.

6 Wolfe DE, Drummond JC. Differential effects of isoflurane/nitrous oxide on posterior tibial somatosensory evoked responses of cortical and subcortical origin. Anesth Analg 1988; 67: 852-9.

7 Kalkman CJ, Traast H, Zuurmond WW, Bovill JG. Differential effects of propofol and nitrous oxide on posterior tibial nerve somatosensory cortical evoked potentials during alfentanil anaesthesia. Br J Anaesth 1991; 66: 483-9.

8 Sloan TB, Ronai AK, Toleikis JR, Koht A. Improvement of intraoperative somatosensory evoked potentials by etomidate. Anesth Analg 1988; 67.

9 Kalkman CJ, Drummond JC, Ribberink AA. Low concentrations of isoflurane abolish motor evoked responses to transcranial electrical stimulation during nitrous oxide/opioid anesthesia in humans. Anesth Analg 1991; 73: 410-5.

10 Kalkman CJ, Drummond JC, Ribberink AA, Patel PM, Sano T, Bickford RG. Effects of propofol, etomidate, midazolam and fentanyl on motor evoked responses to transcranial electrical or magnetic stimulation in humans. Anesthesiology 1992; 76: 502-9.

11 Kalkman CJ, Drummond JC, Kennelly NA, et al. Intraoperative monitoring of tibialis anterior muscle motor evoked responses to transcranial electrical stimulation during partial neuromuscular blockade. Anesth Analg 1992; 75: 584-9.

12 Ubags LH, Kalkman CJ, Been HD. Influence of isoflurane on myogenic motor evoked potentials to single and multiple transcranial stimuli during nitrous oxide/opioid anesthesia. Neurosurgery 1998; 43 : 90-4; discussion 4-5.

13 Nathan N, Tabaraud F, Lacroix F, et al. Influence of propofol concentrations on multipulse transcranial motor evoked potentials. Br J Anaesth 2003; 91: 493-7.

14 Pelosi L, Stevenson M, Hobbs GJ, et al. Intraoperative motor evoked potentials to transcranial electrical stimulation during two anaesthetic regimens. Clin Neurophysiol 2001; 112: 1076-87.

15 Drummond JC, Iragui-Madoz VJ, Alksne JF, Kalkman CJ. Masking of epileptiform activity by propofol during seizure surgery. Anesthesiology 1992; 76: 652-4.

16 Wyler AR, Richey ET, Atkinson RA, Hermann BP. Methohexital activation of epileptogenic foci during acute electrocorticography. Epilepsia 1987; 28: 490-4.

17 Gancher S, Laxer KD, Krieger W. Activation of epileptogenic activity by etomidate. Anesthesiology 1984; 61: 616-8.

18 Keene DL, Roberts D, Splinter WM, et al. Alfentanil mediated activation of epileptiform activity in the electrocorticogram during resection of epileptogenic foci. Can J Neurol Sci 1997; 24: 37-9.

19 Kawaguchi M, Kimbro JR, Drummond JC, et al. Isoflurane delays but does not prevent cerebral infarction in rats subjected to focal ischemia. Anesthesiology 2000; 92: 1335-42.

20 Hoffman WE, Charbel FT, Edelman G, et al. Comparison of the effect of etomidate and desflurane on brain tissue gases and $\mathrm{pH}$ during prolonged middle cerebral artery occlusion. Anesthesiology 1998; 88: 1188-94.

21 Jaaskelainen SK, Kaisti K, Suni L, et al. Sevoflurane is epileptogenic in healthy subjects at surgical levels of anesthesia. Neurology 2003; 61: 1073-8.

22 Jevtovic-Todorovic V, Hartman RE, Izumi $\Upsilon$, et al. Early exposure to common anesthetic agents causes widespread neurodegeneration in the developing rat brain and persistent learning deficits. J Neurosci 2003; 23: 876-82.

23 Olney JW, Young C, Wozniak DF, et al. Anesthesiainduced developmental neuroapoptosis. Does it happen in humans? Anesthesiology 2004; 101: 273-5. 